\title{
Sexo femenino y capacidades matemáticas: desempeño de los más capaces en pruebas de rendimiento matemático
}

Ramón García Perales a

\section{Resumen}

En este artículo se hace un análisis de las diferencias entre sexos en una prueba de rendimiento denominada BECOMA, Batería de Evaluación de la Competencia Matemática, utilizando como marco de referencia las competencias, en este caso, la matemática. Las disciplinas científicas y técnicas son un campo tradicionalmente "masculino", tal y como se observa en múltiples investigaciones en educación, lo que hace imprescindible ahondar en este tema. En principio, se realiza una aproximación a los resultados en PISA, Program for International Student Assessment - esta prueba es elegida por su importancia, el elevado número de países participantes, la solidez y el rigor de los marcos teóricos propuestos y de los análisis efectuados-. Luego, se hace una aproximación al desempeño de los más capaces. Para ello, se establece una relación entre competencia matemática, alto rendimiento (niveles 6 y 7 de desempeño en BECOMA) y sexo femenino. La atención a la diversidad es un principio fundamental de la educación inclusiva $\mathrm{y}$, como tal, la detección de los alumnos con mayor rendimiento en matemáticas resulta imprescindible.

Palabras clave: Diferencias entre sexos. Pruebas de rendimiento. Razonamiento matemático. Diagnóstico de la competencia matemática. Alumnos con alta capacidad matemática.

\footnotetext{
a Junta de Comunidades de Castilla la Mancha, Cuerpo de educación secundaria. Es Castilla La Mancha, España. 


\section{Introducción}

Las diferencias entre sexos pueden tener su origen en cualidades, conductas e identidades determinadas por el proceso de socialización. Estas desigualdades aparecen tanto en las relaciones de poder, como en el acceso a las decisiones y a los recursos. Las posiciones diferentes de los hombres y las mujeres se encuentran influenciadas por realidades históricas, religiosas, económicas, culturales y educativas.

En el terreno educativo, existen investigaciones que consideran que a veces las mujeres son especialmente vulnerables porque no reciben aceptación social por parte de aquellas personas que las rodean: sean padres, amigos o profesores. Además, en ocasiones, reciben mensajes implícitos de infravaloración hacia sus dotes y habilidades. También existen factores como: la época histórica, la edad, la etnicidad, la clase social, la ubicación geográfica, la orientación sexual, la religiosidad, la posición socioeconómica, el patrón de autopercepción y los objetivos de las actividades a las que se enfrentan, que influyen en su desenvolvimiento social $\mathrm{y}$, por en ende, en sus experiencias personales, sean cuales sean sus capacidades (ARNOLD; NOBLE; SUBOTNIK, 1996; GARCÍA PERALES, 2014; HELLER, 1999; JIMÉNEZ FERNANDEZ, 2002; JIMÉNEZ FERNANDEZ et al., 2010; NODA RODRÍGUEZ, 2003; POMAR TOJO et al., 2009).

Se puede manifestar que a lo largo de la escolaridad, chicos y chicas presentan buen desarrollo personal, escolar, familiar y social, aunque manifiestan algunos rasgos diferenciales (JIMÉNEZ FERNANDEZ, 2014). Una realidad que establece rasgos distintivos entre ambos sexos es el papel que juega la inteligencia. En este sentido, Jiménez Fernandez afirma que (2002, p. 76):

"Los más capaces en general, y particularmente las mujeres,
tienen una especial sensibilidad para captar las necesidades ajenas,
especialmente las de los seres próximos más necesitados, y sienten
que deben comprometerse con ellos pudiendo ver desbordadas
sus posibilidades personales, en todo caso, disminuido su
desarrollo personal".

Continuando con la importancia que tiene la consideración de las potencialidades femeninas, Ellis y Willinsky (1999) consideran que en el caso de las mujeres más capaces, puede establecerse una disonancia interna entre lo que son conscientes que pueden llegar a ser, y aquello con lo que se supone que deben conformarse, siguiendo los roles femeninos tradicionales. Eso les provoca, en muchos casos, sentimientos de ansiedad y culpa. Heller (1999) afirma que habitualmente las 
niñas tienden a mostrar con menor frecuencia sus potencialidades, debido a la existencia de roles que valoran más a los chicos que a las chicas, así como a: menor competitividad, mejor trabajo colaborativo, y escasa formación de las mujeres en tareas relacionadas con las matemáticas.

En el campo de las matemáticas, desde edades tempranas se observa que los niños y niñas utilizan procedimientos elementales para buscar solución a situaciones problemáticas de la vida real. Esto permite ver cómo van estructurando su pensamiento matemático, aunque no hayan sido instruidos formalmente en el aprendizaje de reglas y algoritmos convencionales. Todo esto va en consonancia con la finalidad del aprendizaje matemático en el sentido de adquirir un conocimiento ágil, flexible y generalizable a una amplia variedad de situaciones, a las que alumno debe hacer frente, evitando las actitudes de rechazo que a veces esta disciplina genera.

Las matemáticas deberían ser utilizadas como una herramienta más para atender a la diversidad en las aulas, sobre todo cuando nos encontramos con alumnos y alumnas con altas capacidades. Si nos centramos en la capacidad superior matemática, se dice que un alumno tiene talento matemático cuando presenta una capacidad excepcional para las matemáticas, considerándose como un talento simple, o múltiple, si aparece asociado con otros. En ocasiones, este talento puede pasar desapercibido, confundiéndose con un elevado, medio, o bajo rendimiento. Esto provoca desatención, al no ajustar el proceso de enseñanza y aprendizaje a sus potencialidades y, por el contrario, al pasar al estado de aburrimiento, frustración y desinterés, situación que podrá conducir, en algunos casos, a tener fracaso escolar (GARCÍA PERALES, 2014).

Los alumnos con talento matemático muestran desde el inicio de su escolaridad una intensa actividad y un elevado interés hacia los aprendizajes matemáticos, mostrándose curiosos y persistentes hacia este campo. $\mathrm{Su}$ excelente rapidez en la captación de conceptos matemáticos complejos, les permitirá avanzar progresivamente hacia aprendizajes cada vez más abstractos. Según Krutetskii (1976), pionero en el estudio de las características de los niños con talento matemático, los rasgos definitorios de este tipo de talento son:

- Buscan soluciones simples y directas.

- Examinan el contenido matemático de un problema de forma analítica y sintética.

- Investigan aspectos difíciles del problema antes de tratar de resolverlos. 
- Muestran una abreviación de los procesos al resolver problemas de tipo similar; es decir, después de una exposición corta, llegan a considerar ciertos pasos en la resolución como obvios, y usan formas abstractas o abreviadas de razonamiento, omitiendo los pasos intermedios.

- No están sujetos a técnicas de resolución que han tenido éxito en el pasado, y pueden hacer reajustes cuando estas fallan.

- Pueden invertir fácilmente su proceso de pensamiento.

- Se cansan menos trabajando en matemáticas que en otras materias.

- Son flexibles en su pensamiento, y pueden cambiar con facilidad de un proceso cognitivo a otro, incluso si este es cualitativamente diferente.

- Son rápidos en generalizar el contenido y el método de resolución de un problema.

- Tienden a recordar las estructuras generales y abreviadas de los problemas, y sus soluciones.

Mingus y Grassl (1999) completan el perfil dado por Krutetskii, al incluir la voluntad de estar comprometido y centrado en la tarea y la alta creatividad o el pensamiento divergente.

Entre los avances más recientes realizados en este campo del talento matemático, se pueden mencionar los de Reyes-Santander y Karg (2009), que establecen un modelo de talento matemático definido por las siguientes características:

- Productos y resultados originales e innovadores.

- Persistencia y perseverancia en actividades matemáticas que les interesan y de generación metacognitiva.

- Independencia y profundidad en los procesos cognitivos, sobre todo en relación a la inferencia y conexión entre conceptos.

- Dominio de campos del conocimiento matemático: geométrico, algebraico y numérico.

- Conocimiento, rendimiento e implicación en actividades matemáticas.

- Comprensión, en profundidad, de ideas complejas y abstractas.

- Capacidad de generar ideas creativas y avanzadas dentro del área de matemáticas.

- Capacidad de captar y manipular información.

Tourón y Tourón (2006) establecen las siguientes características para el talento matemático: generalización y transferencia, capacidad de abstracción, rapidez de aprendizaje, flexibilidad en los procesos mentales requeridos para la actividad matemática, habilidad para la inversión de los procesos mentales en el razonamiento matemático, pensamiento lógico, memoria matemática, posesión de una estructura mental matemática, y reducción del proceso de razonamiento matemático. Benavides Simon define como rasgos principales de los individuos con talento, 
los siguientes (2008): alta creatividad en la formulación de soluciones a los problemas, compromiso con la tarea, y alto nivel de control en la resolución de las actividades propuestas. Además, considera que "hay diferentes maneras de tener talento matemático, y no podemos hablar de una lista acabada de características asociadas al talento en general, y al talento matemático en particular" (2008, p. 273).

Una vez indicadas las características de los más capaces para las matemáticas, y antes de pasar a tratar el enfoque metodológico, se realiza un acercamiento a la situación social y educativa de las alumnas más capaces. En este sentido, tomando como base la estadística del curso académico 2012/2013, último curso del que se disponen datos en la página web del Ministerio de Educación, Cultura y Deporte, en España el número total de alumnos con alta capacidad en las enseñanzas no universitarias fue de 12.490 escolares, un $0.16 \%$ del total de la población escolarizada en estas enseñanzas. En la Tabla 1 se observa el reparto por Comunidades Autónomas:

Tabla 1. Reparto de alumnado con alta capacidad para el curso académico 2012/2013.

\begin{tabular}{lccc}
\hline \multirow{2}{*}{ CC. AA. } & No. total de alumnos & \multicolumn{2}{c}{ Alta Capacidad } \\
\cline { 3 - 4 } Andalucía & 1.604 .346 & $\mathbf{n}$ & $\%$ \\
Aragón & 212.613 & 3.957 & 0.25 \\
Asturias & 135.968 & 126 & 0.06 \\
Baleares & 176.402 & 435 & 0.32 \\
Canarias & 348.886 & 430 & 0.24 \\
Cantabria & 90.727 & 1.114 & 0.32 \\
Castilla y León & 363.197 & 113 & 0.12 \\
Castilla La Mancha & 376.030 & 499 & 0.14 \\
Cataluña & 1.307 .724 & 239 & 0.06 \\
Com. Valenciana & 850.613 & 233 & 0.02 \\
Extremadura & 184.686 & 189 & 0.02 \\
Galicia & 402.070 & 148 & 0.08 \\
Madrid & 1.127 .255 & 1.057 & 0.26 \\
Murcia & 282.985 & 1.455 & 0.13 \\
Navarra & 104.724 & 1.999 & 0.71 \\
País Vasco & 359.618 & 222 & 0.21 \\
La Rioja & 53.295 & 218 & 0.06 \\
Ceuta & 18.974 & 52 & 0.10 \\
Melilla & 19.243 & 1 & 0.01 \\
España & 8.019 .356 & 3 & 0.02 \\
\hline & & 12.490 & 0.16 \\
\hline
\end{tabular}

Fuente: Elaboración propia (2015). 
De los 12.490 alumnos con alta capacidad en nuestro país, 4.202 eran alumnas, un $33.64 \%$ frente al $66.36 \%$ de los alumnos. En la Tabla 2 se puede observar la tendencia de menor número de casos identificados de alumnas con alta capacidad, tanto a nivel nacional como regional.

Tabla 2. Reparto de alumnado con alta capacidad por sexo para el curso académico 2012/2013.

\begin{tabular}{|c|c|c|c|c|c|}
\hline \multirow{2}{*}{ CC. AA. } & \multirow{2}{*}{$\begin{array}{l}\text { No. total de alumnos } \\
\text { con alta capacidad }\end{array}$} & \multicolumn{2}{|c|}{ Hombres } & \multicolumn{2}{|c|}{ Mujeres } \\
\hline & & $\mathbf{n}$ & $\%$ & $\mathbf{n}$ & $\%$ \\
\hline Andalucía & 3.957 & 2.573 & 65.02 & 1.384 & 34.98 \\
\hline Aragón & 126 & 103 & 81.75 & 23 & 18.25 \\
\hline Asturias & 435 & 283 & 65.06 & 152 & 34.94 \\
\hline Baleares & 430 & 306 & 71.16 & 124 & 28.84 \\
\hline Canarias & 1.114 & 696 & 62.48 & 418 & 37.52 \\
\hline Cantabria & 113 & 83 & 73.45 & 30 & 26.55 \\
\hline Castilla y León & 499 & 349 & 69.94 & 150 & 30.06 \\
\hline Castilla La Mancha & 239 & 158 & 66.11 & 81 & 33.89 \\
\hline Cataluña & 233 & 148 & 63.52 & 85 & 36.48 \\
\hline Com. Valenciana & 189 & 112 & 59.26 & 77 & 40.74 \\
\hline Extremadura & 148 & 119 & 80.41 & 29 & 19.59 \\
\hline Galicia & 1.057 & 712 & 67.36 & 345 & 32.64 \\
\hline Madrid & 1.455 & 1.007 & 69.21 & 448 & 30.79 \\
\hline Murcia & 1.999 & 1.289 & 64.48 & 710 & 35.52 \\
\hline Navarra & 222 & 155 & 69.82 & 67 & 30.18 \\
\hline País Vasco & 218 & 154 & 70.64 & 64 & 29.36 \\
\hline La Rioja & 52 & 38 & 73.08 & 14 & 26.92 \\
\hline Ceuta & 1 & 1 & 100 & 0 & 0 \\
\hline Melilla & 3 & 2 & 66.67 & 1 & 33.33 \\
\hline España & 12.490 & 8.288 & 66.36 & 4.202 & 33.64 \\
\hline
\end{tabular}

Fuente: Elaboración propia (2015).

Como puede observarse, existe una desigualdad, bastante manifiesta, en el diagnóstico, entre sexos. A pesar de ello, desde los años noventa se viene constatando un avance significativo de las mujeres en todas las etapas educativas (JIMÉNEZ y GARCÍA, 2013). Continuando con las estadísticas del curso académico 2012/2013 sobre la escolarización de los alumnos en función de su sexo, aparece que (JIMÉNEZ, 2014): 
En la Figura llama especialmente la atención la diferencia entre las enseñanzas, específicamente entre el ciclo formativos de grado medio -en donde hay un mayor porcentaje de hombres con alta capacidad- y la universidad -en donde las cifras de estudiantes con alta capacidad son superiores en mujeres que en hombres-. Se puede concluir que conforme se avance hacia etapas no obligatorias, el porcentaje de alumnado masculino con alta capacidad disminuye frente al alumnado femenino.

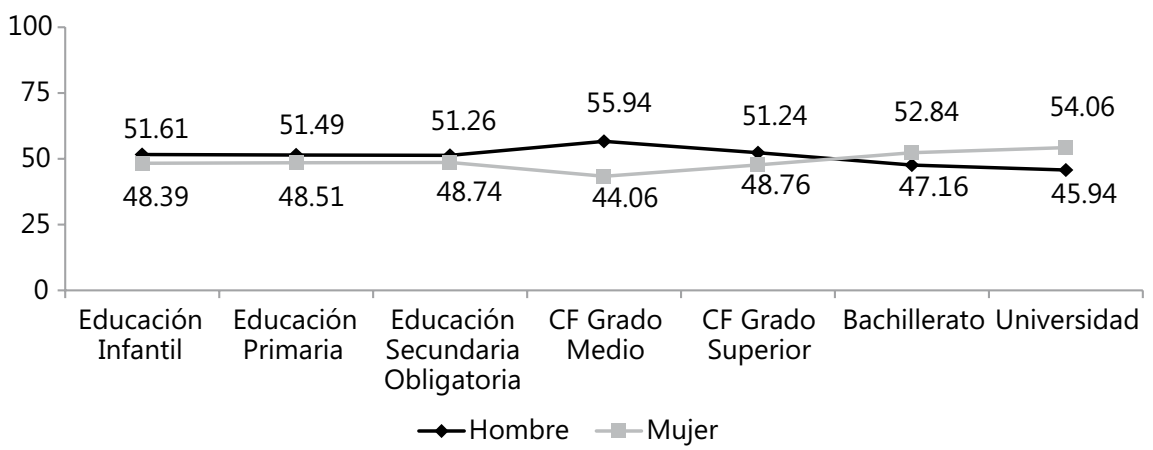

Fuente: Jiménez Fernandez (2014).

Figura. Porcentaje de alumnado escolarizado en todas las enseñanzas en función del sexo.

En este campo de los más capaces, es importante señalar el informe PISA Top of de Class (OCDE, 2009). En él, aparece que el $4.1 \%$ de las alumnas y el 3.9\% de los alumnos son excelentes en lectura, matemáticas y ciencias, al mismo tiempo, y en al menos en una de estas áreas, sobresale el 18.6\% de las alumnas y el 17.3\% de los alumnos (JIMÉNEZ FERNANDEZ; GARCÍA PERALES, 2013). Teniendo en cuenta todas la ediciones de PISA para la competencia matemática, el promedio de España de alumnos con rendimiento excelente es de 7.75\%, mientras que el de la OCDE es de un $13.5 \%$, siendo los resultados de nuestro país inferiores en este campo de rendimiento excelente (GARCÍA PERALES, 2014; JIMÉNEZ FERNANDEZ; BAEZA DELGADO, 2012).

Otros estudios evidencian que no existen diferencias significativas, según sexo, de los alumnos más capaces. Es el caso de los que obtienen Premio Extraordinario de Bachillerato en la Comunidad de Madrid, y Beca de Excelencia para estudiar en las universidades de Madrid (JIMÉNEZ et al., 2005, 2010; JIMÉNEZ y BAEZA, 2012), así como de los resultados obtenidos en el estudio empírico de la tesis doctoral de García Perales (2014). 
Por último, en el caso específico de los más capaces para la matemática, aparecen diferencias estadísticas significativas entre chicos y chicas (INEE, 2010, 2013). Estas diferencias podrían venir derivadas de las distintas experiencias de socialización a las que se enfrenta uno y otro sexo, ya que, en palabras de Pérez Sánchez y Díaz Fernández (1994, p. 110), "los estudios que plantean la hipótesis de un factor hereditario unido al sexo para la aptitud matemática o espacial, o de una especialización lateral diferenciada del cerebro para hombres y mujeres, son muy poco consistentes". A continuación, se hace una aproximación empírica a esta relación entre competencia matemática, alto rendimiento y sexo femenino.

\section{Método}

Tal y como se debería exigir en investigaciones académicas, y con el objetivo de aportar contribuciones destacadas a partir de este artículo, se pretende establecer relaciones entre los datos de una investigación empírica, y el contexto en el que ella se inserta, con la finalidad de profundizar en el análisis e interpretación de datos (JACOMINI, 2014).

Este artículo parte del análisis de los resultados obtenidos en el proceso de construcción y validación de la Batería de Evaluación de la Competencia Matemática (BECOMA). Antes de señalar estos resultados, se realizará un acercamiento a las conclusiones obtenidas en las evaluaciones PISA para la competencia matemática, siendo las ediciones de 2003 y 2012 las que toman esta área de evaluación como prioritaria. PISA consiste en una evaluación estandarizada puesta en marcha a nivel internacional de manera conjunta por los países participantes, desarrollada cada tres años, centrada en evaluación de las áreas de lengua, matemáticas y ciencias, con la finalidad de ofrecer información puntual y, a su vez, longitudinal, para encauzar propuestas de mejora e innovaciones educativas. A pesar de que los alumnos evaluados, sus edades y los momentos de administración son diferentes, entre la BECOMA y PISA, la comparativa entre ambos instrumentos ofrece un punto de vista interesante en la interrelación entre competencia matemática, alto rendimiento y sexo femenino, objetivo principal del presente artículo.

El proceso desarrollado en la construcción y validación de la BECOMA empieza desde el momento en el que se selecciona el constructo a medir, la competencia matemática, y termina en la construcción de una batería de evaluación final compuesta por 34 ítems, repartidos entre 6 factores:

- Factor 1: Sucesiones (6 items). Supone un 17.65\% del contenido de la batería. Se trata de la deducción de una secuencia numérica de términos que 
persiguen un patrón, o regla de formación dada, y en la que cada número tiene un orden relevante a tener en cuenta para su solución.

- Factor 2: Estructuración gráfica (9 items). Engloba un 26.47\% del conjunto del instrumento. Consiste en la interpretación y organización de la información mediante un diseño gráfico, observando posibles regularidades, y valorando su importancia y sus posibilidades de generalización, a otros contenidos del campo de las matemáticas.

- Factor 3: Partes del todo (7 items). Abarca un 20.59\% del conjunto de la batería. Se trata de cálculos aritméticos, a partir de las partes posibles e iguales en las que se pueden diseccionar los números, y las unidades de medida.

- Factor 4: Resolución de problemas (4 items). Supone un 11.76\% del contenido. Engloba la producción y resolución de problemas a partir de datos dados, implicando capacidades mentales superiores, al requerir estrategias diferentes a las exigidas en el planteamiento de problemas de manera tradicional.

- Factor 5: Diez, cien, mil (5 items). Abarca un 14.71\% del contenido de la batería. Incluye la segmentación de números en partes fundamentadas en potencias de base 10, de exponente natural, tomadas como el soporte de la numeración decimal utilizada de manera habitual.

- Factor 6: Descomposición y propiedades (3 items). Engloba un 8.82\% del conjunto del instrumento. Consiste en la organización de un número, según su conveniencia, utilizando distintos contenidos matemáticos, tales como las operaciones y las propiedades asociativa y distributiva.

Con esta prueba se pretende medir los aspectos cuantitativos y cualitativos del rendimiento matemático de los alumnos, en función de siete niveles de desempeño para cada competencia $(1,2,3,4,5,6$ y 7$)$, y a lo largo de 8 pruebas aplicadas de manera colectiva, y definidas de la siguiente manera:

- Prueba 1 Interpretación matemática: consta de 5 ítems sobre contenidos relacionados con la estadística y la probabilidad;

- Prueba 2 Cálculo mental: conformada por 6 ítems de cálculo mental, en los que el alumno debe señalar la solución, intentando no hacer ninguna operación. Cada ítem está compuesto por dos operaciones;

- Prueba 3 Propiedades geométricas: compuesta por 2 ítems de geometría en diferentes formatos;

- Prueba 4 Series lógicas numéricas: incluye 6 ítems en los que el alumno debe averiguar cuál es el elemento que falta al final de cada serie. Cada ítem está compuesto por dos series;

- Prueba 5 Descubriendo algoritmos: consta de 2 ítems en los que el alumno, teniendo unos datos, debe llegar a un resultado dado a través de las operaciones que crea convenientes; 
- Prueba 6 Unidades convencionales: conformada por 6 ítems sobre las distintas unidades de medida, y sobre el uso de los distintos tipos de proporcionalidad existentes;

- Prueba 7 Series lógicas de figuras: abarca 3 ítems en los que el alumno debe dibujar cuál es el elemento que falta al final de cada serie. Cada ítem está compuesto por dos series;

- Prueba 8 Invención de problemas: constituida por 4 ítems en los que, a partir de datos dados, el alumno debe producir un problema en términos de: coherencia global del enunciado, utilización de los datos, redacción adecuada, desarrollo correcto del procedimiento de resolución, y exposición de la solución con su magnitud correspondiente. De los 4 ítems, 2 se centran en la redacción de dos problemas, y el resto en su resolución y en la especificación de la solución.

En cuanto a la fundamentación estadística del instrumento, han aparecido elevados índices: de validez de contenido (la mayoría superiores a .80, siendo el global de .81); de fiabilidad (los índices calculados oscilan entre .73 y .90 , mostrando una consistencia interna alta); de validez de constructo (explorada mediante tres formas: el análisis factorial exploratorio, el estudio de los resultados en función del sexo, y la comparación de las puntuaciones obtenidas con los resultados de los alumnos en las pruebas Series numéricas y Problemas numéricos del BADyG-E3, tomando también como variable el promedio de ambas; a modo de ejemplo, los resultados de correlacionar ambos instrumentos han dado índices de correlación $r$ de Pearson bastante altos y significativos de $.89, .86$ y .85); y de validez de criterio (de tipo concurrente, y comprobada a partir de la relación entre los resultados alcanzados en la batería con los siguientes indicadores de aptitud matemática: rendimiento del alumno en el área de matemáticas; interés del alumno por las matemáticas, de acuerdo al punto de vista del profesor y según el suyo propio; y posible existencia de una elevada aptitud matemática a juicio del tutor. En todas estas variables han aparecido diferencias estadísticamente significativas). Además, el instrumento presenta un equilibrio en cuanto a su dificultad, siendo el índice global de .52, gracias a una amplia variedad de ítems de distinto tipo.

Una vez obtenido este instrumento definitivo, ha sido administrado a 712 alumnos de $5^{\circ}$ de Educación Primaria de diversos centros educativos de la provincia de Albacete, para observar el rendimiento matemático alcanzado. Esta cantidad supone un $17.94 \%$ de la muestra de este nivel en esta provincia, para el curso escolar 2011/2012, comprendiendo a alumnos de 10 años -u 11, en el caso de escolares repetidores-. Las puntuaciones alcanzadas son analizadas en función de distintas variables de estudio, principalmente relacionadas con la competencia matemática, aunque también con otras, pues el sexo de los alumnos es considerado como una variable fundamental. 


\section{Resultados}

Antes de abordar los resultados obtenidos en la batería BECOMA, se indican los resultados en PISA, ejemplo de evaluación internacional de rendimiento, según la variable sexo. En la primera edición de PISA del año 2000, en casi la mitad de los países participantes, se observaron diferencias estadísticamente significativas en matemáticas a favor de los hombres (OCDE, 2002), apuntando que estas diferencias "no son resultado inevitable de las diferencias en estilos de aprendizaje entre hombres y mujeres jóvenes" (OCDE, 2002, p. 135). Además se sugiere que hay "características subyacentes de los sistemas educativos o de las sociedades y las culturas que inciden sobre las diferencias entre géneros en el desempeño a través de la escolaridad" (OCDE, 2002, p. 137).

PISA 2009 justifica la presencia del sexo en los análisis de los resultados "porque permite constatar el posible efecto de las diferentes políticas de igualdad que deben desarrollar los países y las escuelas, a fin de garantizar el máximo desarrollo educativo de todas las personas" (INEE, 2010, p. 106). Ya en PISA 2012 (INEE, 2013), y porque tiene el objetivo de facilitar la presentación y el análisis de los resultados para estos dos subgrupos de alumnos fundamentales.

El análisis de la variable sexo para el área de matemáticas a lo largo de todas las evaluaciones PISA realizadas, muestra que el promedio de la OCDE en matemáticas es siempre superior en los alumnos respecto a las alumnas, oscilando entre los 11 puntos de PISA 2000, 2003, 2006 y 2012 a los 12 puntos de 2009. En solución de problemas, evaluada en PISA 2003 y 2009, el promedio de la OCDE es 2 puntos superior a favor de las mujeres, en la primera de las evaluaciones, y de 7 para los hombres, en la segunda. Todas las puntuaciones promedio de la OCDE muestran diferencias estadísticamente significativas a favor de los alumnos, excepto en solución de problemas, campo en que estas no lo son.

En nuestro país, también se observan resultados similares, siempre a favor de los chicos. Las diferencias van desde los 9 puntos de PISA 2003 y 2006, a los 16 de PISA 2012, y de los 18 de PISA 2000 a los 19 de PISA 2009. En relación a las Comunidades Autónomas, en el ejercicio de sus competencias educativas, eligen si deciden o no participar en PISA, ampliándose la muestra nacional para permitir que sus resultados tengan la precisión estadística suficiente para poder ser comparados entre sí, y con los países participantes en PISA. Los resultados e las regiones españolas en cada edición de PISA fueron los siguientes (GARCÍA PERALES, 2014) (Tabla 3): 
Tabla 3. Resultados según el sexo de los alumnos en las Comunidades Autónomas participantes en PISA.

\begin{tabular}{|c|c|}
\hline 2000 & No participaron las Comunidades Autónomas. \\
\hline 2003 & $\begin{array}{l}\text { Todas las diferencias son a favor de los alumnos ( } 18 \text { puntos por encima en } \\
\text { Cataluña, } 11 \text { en Castilla y León, y } 1 \text { en País Vasco). En solución de problemas, la } \\
\text { diferencia llega a } 6 \text { puntos para las alumnas. Por Comunidades Autónomas, en las } \\
\text { tres regiones los resultados de las alumnas son mejores que los de los alumnos } \\
\text { (12 puntos superior en País Vasco, } 6 \text { en Castilla y León, y } 3 \text { en Cataluña). }\end{array}$ \\
\hline 2006 & $\begin{array}{l}\text { Todas las diferencias son a favor de los alumnos; en el País Vasco la diferencia es } \\
\text { de } 3 \text { puntos; en Cantabria y Asturias de 9; en Navarra y Galicia de 10; Cataluña, } \\
\text { Andalucía y Castilla y León de 11; en La Rioja de 13; en Aragón de 18. }\end{array}$ \\
\hline 2009 & $\begin{array}{l}\text { Todas las comparaciones muestran puntuaciones superiores para los alumnos: en } \\
\text { Andalucía la diferencia es de } 26 \text { puntos; en Cataluña de } 22 \text {; en Baleares de } 21 \text {; en } \\
\text { Aragón y La Rioja, de 19; en Canarias, Cantabria y Murcia, de 17; en Castilla y León, y } \\
\text { Navarra de } 14 ; \text { en Asturias de } 12 ; \text { en Galicia y Madrid de 11; en el País Vasco de } 8 .\end{array}$ \\
\hline 2012 & $\begin{array}{l}\text { Todas señalan diferencias a favor de los hombres. En Cataluña aparece una } \\
\text { diferencia de } 22 \text { puntos; en La Rioja de 19; en Castilla y León de 18; en Aragón } \\
\text { de 17; en Andalucía de 16; en Cantabria y Madrid de 15; en País Vasco de 14; } \\
\text { en Murcia de 13; en Extremadura de 10; en Asturias de 9; en Baleares de 8; en } \\
\text { Navarra de 6; en Galicia de } 2 \text {. En solución de problemas, la diferencia es de } 1.5 \\
\text { puntos para los alumnos. Por Comunidades Autónomas, las diferencias van desde } \\
\text { los } 4 \text { puntos a favor de los alumnos en País Vasco y Madrid, hasta los } 2 \text { puntos } \\
\text { para las alumnas en Cataluña. }\end{array}$ \\
\hline
\end{tabular}

Fuente: Elaboración propia (2014).

Una vez señalados los principales resultados en PISA según esta variable, se muestran los resultados obtenidos en la batería BECOMA, incidiendo en la parte final, es decir, en los resultados de los alumnos más capaces.

\subsection{Resultados según el sexo de los alumnos}

Con el análisis de los resultados en función de esta variable, se ha intentado observar si en las puntuaciones obtenidas aparece una superioridad de un sexo respecto al otro, tal y como se concluye en algunos estudios e investigaciones sobre competencia matemática (INEE, 2010, 2013).

Siguiendo la estadística del Ministerio de Educación, Cultura y Deporte, en el curso escolar 2011/2012, momento de referencia del estudio, el porcentaje de alumnos escolarizados en $5^{\circ}$ de Educación Primaria en España era de 51.27\%, y el de alumnas era de $48.73 \%$. En Castilla La Mancha, para el mismo curso académico, los porcentajes eran de $51.45 \%$ y $48.55 \%$, respectivamente, mientras que en la provincia de Albacete, de $50.51 \%$ y $49.49 \%$. 
En la Tabla 4 se puede ver la distribución de los alumnos, según su sexo, dentro del total de la muestra participante en la investigación:

Tabla 4. Distribución por sexos de la muestra participante.

\begin{tabular}{cccc}
\hline & & $\boldsymbol{n}$ & $\%$ \\
\hline Sexo & Hombre & 360 & 50.56 \\
& Mujer & 352 & 49.44 \\
\hline & Total & 712 & 100 \\
\hline
\end{tabular}

Fuente: Elaboración propia (2014).

La puntuación media total en la batería, para los alumnos, ha sido de 35.54 $(D T=13.60)$, y para las alumnas, de $31.85(D T=12.54)$. Tras un análisis detallado de las puntuaciones, en función del sexo, se han observado diferencias estadísticamente significativas entre hombres y mujeres (Tabla 5). A favor de los alumnos, en el Total de la Batería, en los factores Sucesiones y Partes del todo y en los ítems 13, 14, 15, 16, 17, 18, 19, 20, 22, 23, 24, 25, 26, 27 y 28. Solo en el ítem 8 han sido a favor de las alumnas.

En los factores Resolución de problemas y Descomposición y propiedades, y en los ítems 3, 4, 5, 7, 8, 11, 30, 31 y 33, las puntuaciones de las alumnas han sido superiores a las de los alumnos, alcanzando únicamente la significación estadística en el ítem 8 .

Tabla 5. Prueba t para muestras independientes en función del sexo.

\begin{tabular}{|c|c|c|c|c|c|c|c|}
\hline & \multicolumn{2}{|c|}{ Hombre } & \multicolumn{2}{|c|}{ Mujer } & \multirow{2}{*}{$\mathbf{t}$} & \multirow{2}{*}{ gl } & \multirow{2}{*}{$\mathbf{p}$} \\
\hline & M & DT & M & DT & & & \\
\hline \multicolumn{8}{|c|}{ F1: Sucesiones } \\
\hline IT 14 & 1.73 & .52 & 1.62 & .57 & 2.77 & 710 & $.006^{* *}$ \\
\hline IT 15 & 1.33 & .72 & 1.03 & .73 & 5.50 & 710 & $.000^{* *}$ \\
\hline IT 16 & 1.14 & .82 & .83 & .76 & 5.30 & 710 & $.000 * *$ \\
\hline IT 17 & 1.19 & .79 & .98 & .73 & 3.80 & 710 & $.000 * *$ \\
\hline IT 18 & 1.07 & .70 & .87 & .73 & 3.83 & 710 & $.000^{* *}$ \\
\hline IT 19 & .96 & .70 & .65 & .63 & 6.19 & 710 & $.000 * *$ \\
\hline Total Factor & 7.44 & 3.00 & 5.97 & 2.68 & 6.85 & 710 & $.000^{* *}$ \\
\hline
\end{tabular}

Continúa 
Continuación

F2: Estructuración gráfica

\begin{tabular}{cccccccc} 
IT 1 & .91 & .99 & .83 & .97 & 1.07 & 710 & .285 \\
IT 2 & .51 & .86 & .39 & .78 & 1.93 & 710 & .053 \\
IT 3 & 1.07 & .95 & 1.11 & .96 & -.66 & 710 & .512 \\
IT 4 & 1.22 & .86 & 1.23 & .86 & -.12 & 710 & .902 \\
IT 12 & 1.82 & .56 & 1.79 & .58 & .56 & 710 & .575 \\
IT 13 & .94 & .98 & .77 & .96 & 2.32 & 710 & $.021^{*}$ \\
IT 28 & .36 & .62 & .24 & .51 & 2.95 & 710 & $.003^{* *}$ \\
IT 29 & 1.13 & .810 & 1.05 & .78 & 1.43 & 710 & .153 \\
IT 30 & 1.30 & .77 & 1.31 & .78 & -.21 & 710 & .829 \\
\hline Total Factor & 9.25 & 4.15 & 8.72 & 4.09 & 1.73 & 710 & .084 \\
\hline
\end{tabular}

F3: Partes del todo

\begin{tabular}{cccccccc} 
IT 20 & .98 & .99 & .66 & .93 & 4.35 & 710 & $.000^{* *}$ \\
IT 21 & .44 & .82 & .35 & .74 & 1.58 & 710 & .114 \\
IT 22 & 1.17 & .98 & .84 & .98 & 4.51 & 710 & $.000^{* *}$ \\
IT 23 & 1.02 & .94 & .85 & .91 & 2.50 & 710 & $.013^{*}$ \\
IT 24 & .80 & .88 & .60 & .83 & 3.16 & 710 & $.002^{* *}$ \\
IT 25 & .89 & .90 & .66 & .82 & 3.50 & 710 & $.000^{* *}$ \\
IT 26 & .68 & .81 & .46 & .74 & 3.73 & 710 & $.000^{* *}$ \\
\hline Total Factor & 5.97 & 4.03 & 4.41 & 3.73 & 5.35 & 710 & $.000^{* *}$ \\
\hline F4: Resolución de problemas & & & & & & & \\
IT 31 & 1.45 & .84 & 1.53 & .78 & -1.29 & 710 & .196 \\
IT 32 & 1.07 & .95 & 1.06 & .95 & .10 & 710 & .922 \\
IT 33 & .84 & .93 & .85 & .93 & -.03 & 710 & .975 \\
IT 34 & .47 & .79 & .45 & .79 & .25 & 710 & .800 \\
\hline Total Factor & 3.83 & 2.57 & 3.89 & 2.40 & -.31 & 710 & .753 \\
\hline Contin & & & & & & &
\end{tabular}

Continúa 
Continuación

\begin{tabular}{lccccccc}
\hline F5: Diez, cien, mil & & & & & & & \\
IT 5 & 1.30 & .86 & 1.35 & .84 & -.78 & 710 & .437 \\
IT 9 & .93 & .74 & .88 & .73 & .95 & 710 & .340 \\
IT 10 & 1.01 & .77 & .98 & .75 & .45 & 710 & .656 \\
IT 11 & .63 & .70 & .66 & .72 & -.75 & 710 & .452 \\
IT 27 & .94 & .99 & .63 & .92 & 4.39 & 710 & $.000^{* *}$ \\
\hline Total Factor & 4.80 & 2.46 & 4.50 & 2.40 & 1.66 & 710 & .096 \\
\hline F6: Descomposición y propiedades & & & & & & \\
IT 6 & 1.54 & .64 & 1.48 & .67 & 1.19 & 710 & .234 \\
IT 7 & 1.54 & .65 & 1.59 & .60 & -1.17 & 710 & .243 \\
IT 8 & 1.16 & .81 & 1.28 & .74 & -1.97 & 710 & $.049 *$ \\
\hline Total Factor & 4.24 & 1.68 & 4.36 & 1.59 & -.90 & 710 & .367 \\
\hline Total Batería & 35.54 & 13.60 & 31.85 & 12.54 & 3.76 & 710 & $.000^{\star *}$ \\
\hline
\end{tabular}

* Significativa al $5 \%(p<.05)$.

** Significativa al $1 \%(p<.01)$.

Fuente: Elaboración propia (2014).

Como puede observarse, aparecen disparidades entre ambos sexos. Estas desigualdades a favor de los hombres en el rendimiento matemático, se observan en otros muchos estudios e investigaciones (BENNETT, 1996, 1997; CHAN, 2001, 2006; FURNHAM; CLARK; BAILEY, 1999; INEE, 2008, 2010, 2011, 2013; LLOR et al., 2012; PASARÍN VÁZQUEZ et al., 2004; SÁNCHEZ et al., 2008; STANLEY; BENBOW, 1983). De forma complementaria, otras investigaciones reflejan que las más perjudicados en los procesos de diagnóstico de la alta capacidad y el talento son las chicas (KERR, 2000; LANDAU, 2003). Estas conclusiones podrían servir de referencia para que las administraciones educativas establezcan programas específicos, o recursos educativos adicionales para determinados alumnos, tal y como se aconseja desde diferentes marcos nacionales e internacionales (INEE, 2003).

En la actualidad, el reparto de los alumnos en niveles de rendimiento dentro del área de matemáticas es algo común en múltiples investigaciones (RODERER; ROEBERS, 2013). Estos niveles muestran el porcentaje de alumnos que se 
encuentran en cada uno de ellos, y vienen concretados con una descripción de lo que conocen y saben hacer los alumnos que hacen parte de cada uno de ellos. Tanto en PISA como en la batería BECOMA, son siete los niveles de rendimiento fijados, por debajo del nivel 1, 1, 2, 3, 4, 5 y 6, y, 1, 2, 3, 4, 5, 6 y 7 , respectivamente. La finalidad de la definición de estos niveles, es dar mayor sentido educativo a la dispersión de las puntuaciones, mostrando la proporción de sujetos encuadrados en los niveles: bajos, medios y superiores.

\subsection{Rendimiento matemático de los alumnos más capaces}

Los niveles de rendimiento 6 y 7 de la BECOMA son los más elevados (ver Tabla 6) y serán utilizados para el análisis del rendimiento matemático de los alumnos más capaces, de acuerdo con la variable sexo.

Tabla 6. Niveles de rendimiento de la BECOMA.

\begin{tabular}{lccccc}
\hline Niveles & Intervalos & $\mathbf{f}$ & \% & \% válido & \% acumulado \\
\hline 1 & $\leq 8$ & 14 & 2.0 & 2.0 & 2.0 \\
2 & $9-18$ & 88 & 12.4 & 12.4 & 14.3 \\
3 & $19-28$ & 165 & 23.2 & 23.2 & 37.5 \\
4 & $29-38$ & 184 & 25.8 & 25.8 & 63.3 \\
5 & $39-48$ & 159 & 22.3 & 22.3 & 85.7 \\
6 & $49-58$ & 80 & 11.2 & 11.2 & 96.9 \\
7 & $59-68$ & 22 & 3.1 & 3.1 & 100.0 \\
\hline
\end{tabular}

Fuente: Elaboración propia (2014).

En el nivel de rendimiento 6, con puntuaciones entre 49 y 58, aparecen 80 alumnos, un $11.2 \%$ de la muestra. De ello,s 55 han sido hombres y 25 mujeres. El número de alumnos es muy superior al de alumnas, representando cada grupo un $68.75 \%$ y un $31.25 \%$, respectivamente. En muchos ítems y factores, los alumnos han obtenido puntuaciones medias superiores a las de las alumnas. La muestra participante ha sido de $50.6 \%$ de hombres y $49.4 \%$ mujeres, quedando manifiestas las diferencias entre sexos. Tras la prueba de bondad de ajuste ji-cuadrado, aparecen diferencias estadísticamente significativas entre sexos, a favor de los hombres en este nivel.

Ya en el nivel de rendimiento 7, el más alto de la escala, correspondiente a una puntuación directa igual o superior a 59, han aparecido 22 alumnos, un $3.1 \%$ de la muestra. De estos alumnos, 14 han sido hombres y 8 mujeres. El número de alumnos es superior al de alumnas, representado por un $63.64 \% \mathrm{y}$ 
$36.36 \%$, respectivamente. En estas puntuaciones, entre sexos se observa una discrepancia, tras compararlas con la muestra participante, $50.6 \%$ de hombres y $49.4 \%$ de mujeres. De acuerdo a la prueba de bondad de ajuste ji-cuadrado, no existen diferencias estadísticamente significativas entre sexos en el nivel 7 de rendimiento matemático. Esta equidad en los resultados entre los hombres y mujeres más capaces, se observa en otros estudios e investigaciones, como: el informe Top of de Class (OCDE, 2009), los estudiantes que obtienen Premio Extraordinario de Bachillerato, y aquellos que ganan la Beca de Excelencia (JIMÉNEZ FERNANDEZ et al., 2005, 2010).

Este resultado en el nivel de rendimiento 7 no coincide con lo observado en el resto de conclusiones, una vez que al poner en relación las puntuaciones obtenidas de la batería con el sexo de los alumnos, los resultados de los hombres han sido significativamente superiores a los de las mujeres.

En los niveles más elevados de rendimiento de la batería, aparecen un total de 102 alumnos (14.3\% de la muestra participante), 80 alumnos en el nivel 6, más 22 del nivel 7. En PISA 2012, en los dos niveles superiores de rendimiento, niveles 5 y 6 , aparece un $12.6 \%$ de alumnos como promedio de la OCDE (9.3\% y $3.3 \%$, respectivamente), mientras que en España un $8 \%$ (6.7\% y $1.3 \%$, respectivamente). Como puede observarse, el porcentaje de alumnos en los dos niveles de rendimiento superiores de la batería, ha sido mayor al promedio de la OCDE y al de nuestro país en PISA.

\section{Conclusión}

En la actual sociedad del conocimiento, el capital humano constituye la base social, económica y cultural de desarrollo de un país. Debido a ello, los sistemas educativos de los países deberán favorecer una educación más equitativa, que promueva y dé la oportunidad a todos los escolares de demostrar sus potencialidades, otorgando un papel protagonista a aquellos alumnos que cuentan con un talento superior (HERNÁNDEZ TORRANO; GUTIÉRREZ SÁNCHEZ, 2014; JIMÉNEZ FERNANDEZ, 2014; MANDELMAN et al., 2010).

Una de las desigualdades existentes entre hombres y mujeres, hace referencia al diagnóstico de los más capaces. El número de casos detectados con alta capacidad en los centros educativos es reducido, quedando todavía camino por recorrer. En el caso de las mujeres, su prevalencia entre los más capaces es significativamente más baja respecto a la de los chicos. Esto deriva en su consideración como grupo de riesgo debido a la complejidad de su diferenciación, apareciendo una mayor probabilidad de vulnerabilidad y fracaso escolar. 
En el caso de la capacidad superior matemática, la situación de la mujer precisa de una atención prioritaria, ya que existen diversos estudios e investigaciones que concluyen diferencias en razonamiento científico y matemático a favor de los hombres (BARBERO GARCÍA et al., 2007; BELTRÁN; PÉREZ, 1994; FOX; DENHAM, 1974; JIMÉNEZ FERNANDEZ, 2002; PASARÍN VÁZQUEZ et al., 2004; PÉREZ SÁNCHEZ; DOMÍNGUEZ RODRIGUEZ, 2000; SUBERVIOLA OVEJAS, 2012). Desde los centros educativos, se deberá continuar favoreciendo el desarrollo de prácticas coeducativas, con la finalidad de buscar una igualdad de oportunidades real, incidiendo en la necesidad de detectar y responder educativamente a la alta capacidad femenina, y prestando una especial atención a las posibles discrepancias en los resultados en el campo de la matemática. Para ello, se intentará evitar cualquier posible tratamiento discriminatorio que, en ocasiones, pervive, procedente de docentes, de compañeros y de la propia familia (JIMÉNEZ FERNANDEZ et al., 2010).

En las evaluaciones PISA, para la competencia matemática siempre aparecen diferencias a favor de los chicos, respecto a las chicas, tanto en los promedios de la OCDE, como en los resultados globales de España, y en los específicos de cada Comunidad Autónoma. En solución de problemas, campo evaluado en PISA 2003 y 2009, las diferencias son a favor de las mujeres, en la primera edición, y principalmente a favor de los hombres, en la segunda. Con los resultados de las evaluaciones PISA, se busca generar políticas educativas que repercutan en la mejoría de los procesos de enseñanza y aprendizaje de los alumnos, remarcando la importancia de la conexión entre dichas políticas, y la evolución de los indicadores educacionales (OLIVEIRA, 2014).

Esta finalidad también se persigue con la administración de la BECOMA. Tras su aplicación, la puntuación media total para los alumnos ha sido de 35.54 $(D T=13.60)$ y para las alumnas de $31.85(D T=12.54)$. Una vez realizado un análisis detallado de las puntuaciones en función de la variable sexo, se han observado diferencias estadísticamente significativas a favor de los hombres, principalmente. En el caso de los alumnos más capaces, ubicados en los niveles de rendimiento 6 y 7 de la batería, tras la prueba de bondad de ajuste ji-cuadrado, aparecen diferencias estadísticamente significativas entre sexos, a favor de los hombres, en el nivel 6 de rendimiento matemático; sin embargo, en el 7 no existen diferencias entre sexos.

De esta manera, desde una perspectiva educativa, para estos alumnos sería aconsejable la ampliación de tareas y contenidos matemáticos con distintos niveles de dificultad, que lleven a una respuesta educativa lo más ajustada posible. 
Esta intervención específica incluiría contenidos de otras competencias, con la finalidad de favorecer un desarrollo integral del alumnado, atendiendo a sus potencialidades, e incluyendo la búsqueda de una igualdad de oportunidades real entre todos los alumnos. En esta tarea, el profesorado deberá ser consciente de que a veces aparecen problemas de rendimiento, no debidos a una deficiente actitud por parte del alumno, sino más bien a un problema de "aptitud", es decir, a un desnivel en sus capacidades o discrepancia, entre lo que hace y lo que podría ser capaz de hacer, cualquiera sea su sexo.

Todo esto parte de la consideración de que contamos con un sistema educativo que valora positivamente la existencia de la diversidad de sus alumnos. Entre ellos, se encuentran los alumnos más capaces, como un colectivo más que precisa y demanda una respuesta educativa individualizada ajustada a sus potencialidades y sus características singulares, precisando la población femenina una atención específica desde los centros educativos. Por diversas razones, en muchos países está emergiendo el interés por los alumnos más capaces para la matemática, quedando patente la importancia de considerar la variable sexo en ellos. Además, aparece la necesidad de estructurar nuevos programas educativos para el alumnado, en función de sus potencialidades, y sin que su sexo pueda ser un factor influyente en su participación. 


\section{Sexo feminino e capacidades matemáticas: desempenho dos mais capazes em testes de rendimento matemático}

\section{Resumo}

Neste artigo faz-se uma abordagem das diferenças entre sexo em uma prova de rendimento denominada Bateria de Avaliação da Competição Matemática - BECOMA, utilizando como ponto de referência as competências em matemática. As disciplinas científicas e técnicas são um campo tradicionalmente "masculino", tal como se observa em variadas pesquisas em educação, tornando imprescindivel abordar esta temática. De modo prévio, faz-se uma aproximação com os resultados do PISA (Program for International Student Assessment) por causa de sua importância relativa ao grande número de países participantes, a sua solidez, ao rigor dos marcos teóricos propostos e às análises realizadas. Em última instância, faz-se uma aproximação ao desempenho dos mais capazes. Para tal, estabeleceu-se uma relação entre competição matemática, alto rendimento e sexo feminino, usando a bateria BECOMA e os dois primeiros niveis de desempenho, 6 e 7. A atenção para a diversidade é um princípio fundamental da educação inclusiva e, como tal, a descoberta dos estudantes com maior rendimento em matemática é indispensável.

Palavras-chave: Diferenças entre os sexos. Teste de desempenho. Raciocínio matemático. Diagnóstico de competência matemática. Estudantes com alta capacidade matemática.

\section{Female and mathematics skills: best ranked students in tests of mathematical performance}

\section{Abstract}

In this article differences between female and male sexes, in terms of performance in BECOMA Battery of Evaluation of the Mathematical Competition, are analyzed, using the Competencies - especially the mathematical competency-as a framework. Scientific and technical disciplines have traditionally been a "male" field, according to multiple investigations in education; that is why it is indispensable to deepen in this topic. First, a study of PISA Program for International Student Assessment-this test is chosen because of its importance, high number of participant countries, solidity and rigor in evaluation and analysis-, is carried out. Then, an inquiry of the best ranked students is conducted, by establishing a relationship between mathematics skills, high performance (level 6 and 7 in BECOMA) and female sex. Attention to the diversity is a fundamental principle of inclusive education, and in that order, the detection of students with the best mathematics performances is indispensable.

Keywords: Differences among sexes. Yield tests. Mathematical reasoning. Diagnosis of the mathematical competition. Students with high mathematical capacity. 


\section{Referencias}

ARNOLD, K. D.; NOBLE, K. D.; SUBOTNIK, R. F. Remarkable women: perspectives on female talent development. Cresskill, NJ: Hampton, 1996.

BARBERO GARCÍA, M. I. et al. Actitudes, hábitos de estudio y rendimiento en Matemáticas: diferencias por género. Psicothema, Asturias, v. 19, n. 3, p. 413-21, jul. 2007.

BELTRÁN, J. A.; PÉREZ, L. Estudio experimental del autoconcepto académico en alumnos de altas capacidades a través de la escala E.D.D.A. Madrid: Universidad Complutense, 1994.

BENAVIDES SIMON, M. Caracterización de sujetos con talento en resolución de problemas de estructura multiplicativa. 2008. 355 p. Tesis (Doctorado en Didáctica de la Matemática) - Universidad de Granada, Granada, 2008.

BENNETT, M. Men's and women's self-estimates of intelligence. Journal of Social Psychology, London, v. 136, n. 3, p. 411-2, jun. 1996. doi:10.1080/00224545.1996.9714021

BENNETT, M. Self-estimates of ability in men and women. Journal of Social Psychology, London, v. 137, n. 4, p. 540-1, ago. 1997. doi:10.1080/00224549709595475

CHAN, D. W. Assessing giftedness of Chinese secondary students in Hong Kong: a multiple intelligences perspective. High Ability Studies, Oxford, v. 12, n. 2, p. 215-34, dez. 2001. doi:10.1080/13598130120084348

. Perceived multiple intelligences among male and female Chinese gifted students in Hong Kong: the structure of the student multiple intelligences profile. Gifted Child Quarterly, Storrs, v. 50, n. 4, p. 325-38, out. 2006. doi:10.1177/001698620605000405

ELLIS, J.; WILLINSKY, J. Niñas, mujeres y superdotación: un desafío a la discriminación educativa de las mujeres. Madrid: Narcea, 1999.

FOX, L. H.; DENHAM, S.A. Values and career interests of mathematically and scientifically precocious youth. Baltimore, MD: The Johns Hopkins University Press, 1974. 
FURNHAM, A.; CLARK, K.; BAILEY, K. Sex differences in estimates of multiple intelligences. European Journal of Personality, Hoboken, v. 13, n. 4, p. 247-59, aug. 1999. doi:10.1002/(SICI)1099-0984(199907/08)13:4<247::A ID-PER329>3.0.CO;2-7

GARCÍA PERALES, R. Diseño y validación de un instrumento de evaluación de la competencia matemática: rendimiento matemático de los alumnos más capaces. 2014. 414 p. Tesis (Doctorado en Educación) - Universidad Nacional de Educación a Distancia, Madrid, 2014.

HELLER, K. A. Individual learning and motivation. Needs versus instructional conditions of gifted education. High Ability Studies, Oxford, v. 10, n. 1, p. 9-21, jun. 1999.

HERNÁNDEZ TORRANO, D.; GUTIÉRREZ SÁNCHEZ, M. El estudio de la alta capacidad intelectual en España: Análisis de la situación actual. Revista de Educación, Madrid, v. 364, p. 251-72, abr. 2014.

INEE - INSTITUTO NACIONAL DE EVALUACIÓN EDUCATIVA. Marcos teóricos de PISA 2003: Conocimientos y destrezas en Matemáticas, Lectura, Ciencias y Solución de problemas. Madrid: Ministerio de Educación, Cultura y Deporte, 2003.

. PISA 2003: matemáticas: informe español. Madrid: Ministerio de Educación, Cultura y Deporte, 2008.

. PISA 2009: informe Español. Madrid: Ministerio de Educación, Cultura y Deporte, 2010.

. PISA 2012: informe español. Madrid: Ministerio de Educación, Cultura y Deporte, 2013. Volumen I: Resultados y contexto.

. TIMSS 2007: guía del usuario para la base de datos internacional. Madrid: Ministerio de Educación, Cultura y Deporte, 2011.

JACOMINI, M. A. Avaliação da aprendizagem em tempos de progressão continuada: o que mudou? Um estudo de teses e dissertações sobre o tema (2000-2010). Ensaio: Avaliação e Políticas Públicas em Educação, Rio de Janeiro, v. 22, n. 84, p. 807-28, jul. 2014. doi:10.1590/S0104-40362014000300010 
JIMÉNEZ FERNANDEZ, C. Educación, alta capacidad y género: el necesario compromiso entre hombres y mujeres más capaces. Revista del Ministerio de Trabajo y Asuntos Sociales, Madrid, v. 40, n. 12, p. 69-83, 2002.

. El desarrollo del talento: educación y alta capacidad: lección inaugural. Madrid: UNED, 2014. Disponible en: $<$ https://canal.uned.es/ mmobj/index/id/21559>. Acceso en: 15 nov. 2014.

JIMÉNEZ FERNANDEZ, C. et. al. Hacia un modelo sociocultural explicativo del alto rendimiento y la alta capacidad: ámbito académico y capacidades personales. Revista Educación XX1, Madrid, v. 13, n. 1, p. 125-53, jan. 2010.

. Educación, capacidad y género: alumnos con premio extraordinario de Bachillerato. Revista de Investigación Educativa, Murcia, v. 23, n. 2, p. 391-416, jun. 2005.

JIMÉNEZ FERNANDEZ, C.; BAEZA DELGADO, M. A. Factores significativos del rendimiento excelente: PISA y otros estudios. Ensaio: Avaliação e Políticas Públicas em Educação, Rio de Janeiro, v. 20, n. 77, p. 647-77, out. 2012. doi:10.1590/S0104-40362012000400003

JIMÉNEZ FERNANDEZ, C.; GARCÍA PERALES, R. Los alumnos más capaces en España: normativa e incidencia en el diagnóstico y la educación. Revista Española de Orientación y Psicopedagogía, Madrid, v. 24, n. 1, p. 7-24, mar. 2013. doi:10.5944/reop.vol.24.num.1.2013.11267

KERR, B. Guiding gifted girls and young women. In Heller, K. A. et al. International handbook of giftedness and talent. 2nd ed. Oxford, UK: Elsevier, 2000. p. 649-57.

KRUTESKII, V. A. The psychology of mathematical abilities in school children. Chicago: University of Chicago Press, 1976.

LANDAU, E. El valor de ser superdotado. Madrid: Consejería de Educación, 2003.

LLOR, L. et al. Inteligencias múltiples y alta habilidad. Aula Abierta, Oviedo, v. 40, n. 1, p. 27-38, jan. 2012.

MANDELMAN, S. D. et al. Intelectual gifteness: economic, political, cultural and psychological considerations. Learning and Individual Differences, Connecticut, v. 20, p. 286-97, ago. 2010. 
MINGUS, T.; GRASSL, R. What constitutes a nurturing environment for growth of mathematically gifted students? School Science and Mathematics, Hoboken, v. 99, n. 6, p. 286-92, out. 1999.

NODA RODRÍGUEZ, M. M. Sobredotación, test de inteligencia e igualdad de oportunidades educativas: un apunte sociológico. Revista Iberoamericana de Educación, Madrid, p. 1-12, fev. 2003.

OCDE - ORGANIZACIÓN PARA LA COOPERACIÓN Y EL DESARROLLO ECONÓMICO. Conocimiento y aptitudes para la vida: primeros resultados del programa internacional de evaluación de estudiantes (PISA) 2000 de la OCDE. México: Santillana, 2002.

. Top of the class. High performers in science in PISA 2006. París: OCDE, 2009. Disponible en: <http://www.oecd.org/dataoecd/44/17/42645389. pdf>. Acceso en: 12 jul. 2013.

OLIVEIRA, A. G. Políticas educacionais e a qualidade da educação nos municípios fluminenses: alguns recortes. Ensaio: Avaliação e Políticas Públicas em Educação, Rio de Janeiro, v. 22, n. 83, p. 411-42, abr. 2014. doi:10.1590/S0104-40362014000200007

PASARÍN VÁZQUEZ, M. et al. Evaluación del talento matemático en Educación Secundaria. Faisca. Revista de Altas Capacidades, Madrid, n. 11, p. 83-102, 2004.

PÉREZ SÁNCHEZ, L.; DÍAZ FERNÁNDEZ, O. Bajo rendimiento académico y desintegración escolar en alumnos de altas capacidades. Faisca. Revista de Altas Capacidades, Madrid, n. 1, p. 103-27, 1994.

PÉREZ SÁNCHEZ, L.; DOMÍNGUEZ RODRIGUEZ, P. Superdotación y adolescencia: características y necesidades en la comunidad de Madrid. Madrid: Consejería de Educación, Dirección General de Promoción Educativa, 2000.

POMAR TOJO, C. et. al. Habilidades matemáticas y verbales: Diferencias de género en una muestra de $6^{\circ}$ de Primaria y $1^{\circ}$ de ESO. Faisca. Revista de Altas Capacidades, Madrid, v. 14, n. 16, p. 14-26, 2009.

REYES-SANTANDER, P.; KARG, A. Una aproximación al trabajo con niños especialmente dotados en Matemáticas. In GONZÁLEZ LÓPEZ, M. J. et al. (Eds.). Investigación en Educación Matemática XIII. Santander: SEIEM, 2009. p. 403-14. 
RODERER, T.; ROEBERS, C. Children's performance estimation in mathematics and science tests over a school year: a pilot study. Electronic Journal of Research in Educational Psychology, Almería, v. 11, n. 1, p. 5-24, abr. 2013.

SÁNCHEZ, C. et. al. Inteligencias Múltiples y Superdotación. Sobredotação, Braga, 9, 87-105, 2008.

STANLEY, J. C.; BENBOW, C. P. SMPY' first decade: ten years of posing problems and solving them. Journal of Special Education, Austin, v. 17, n. 1, p. 11-25, apr. 1983.

SUBERVIOLA OVEJAS, I. Coeducación: un derecho y un deber del profesorado. Revista Electrónica Interuniversitaria de Formación del Profesorado, Zaragoza, v. 15, n. 3, p. 59-67, out. 2012.

TOURÓN, J.; TOURÓN, M. La identificación del talento verbal y matemático de los jóvenes más capaces: el modelo de CTY España. In: SIMPOSIO INTERNACIONAL SOBRE ALTAS CAPACIDADES, 1., 2006, Las Palmas de Gran Canaria. Las Palmas de Gran Canaria: Consejería de Educación, 2006.

\section{Informação do autor}

Ramón García Perales: Professor del Departamento de Pedagogía de la Facultad de Educación de Albacete de la Universidad de Castilla La Mancha (UCLM), España. Contato: ramongarciaperales@hotmail.com 